\title{
The influence of location and visual features on visual object memory
}

\author{
HSin-Mei Sun ANd Robert D. Gordon \\ North Dakota State University, Fargo, North Dakota
}

\begin{abstract}
In five experiments, we examined the influence of contextual objects' location and visual features on visual memory. Participants' visual memory was tested with a change detection task in which they had to judge whether the orientation (Experiments 1A, 1B, and 2) or color (Experiments 3A and 3B) of a target object was the same. Furthermore, contextual objects' locations and visual features were manipulated in the test image. The results showed that change detection performance was better when contextual objects' locations remained the same from study to test, demonstrating that the original spatial configuration is important for subsequent visual memory retrieval. The results further showed that changes to contextual objects' orientation, but not color, reduced orientation change detection performance; and changes to contextual objects' color, but not orientation, impaired color change detection performance. Therefore, contextual objects' visual features are capable of affecting visual memory. However, selective attention plays an influential role in modulating such effects.
\end{abstract}

Recent research has shown that visual object representations can be maintained reliably during and after a participant's viewing of complex visual stimuli, such as scenes and object arrays (Hollingworth, 2004, 2005; Hollingworth \& Henderson, 2002; Hollingworth, Williams, $\&$ Henderson, 2001; Sun \& Gordon, 2009). For example, Hollingworth et al. tested whether detailed visual representations can be maintained after attention is withdrawn from individual objects in scenes. Participants were asked to detect changes to the visual form of individual objects appearing within line drawings of natural scenes. During free viewing of a scene, a target object was replaced by another object from the same basic-level category during a saccade away from the target object after it had been fixated the first time. The results showed that the participants could successfully detect a token change when the change occurred during a saccade away from the target object, demonstrating that relatively detailed visual representations can be retained in memory from previously attended objects and support the detection of changes to the visual form of those objects. By using the same saccade-contingent change paradigm, Hollingworth and Henderson further demonstrated that participants were able to successfully detect type, token, and rotation changes to a target object when it was no longer within the focus of current attention. In addition, Hollingworth and Henderson administered a delayed memory test and showed that their participants were still able to successfully discriminate targets from distractors after all scenes had been viewed (our Experiments 1A, 1B, and 2). In other words, the participants' discrimination performance in the type, token, and orientation discrimination conditions was well above chance despite the fact that they were tested after a 5- to 30-min retention interval. This finding provided converging evidence that relatively detailed visual information can be retained in memory. Moreover, Hollingworth (2005) had participants view various images of scenes and tested their memory for the visual form of individual objects in the scenes after various retention intervals. The results showed that there was little or no decline in change detection performance from immediate test to a delay of one trial or until the end of the entire study session. Surprisingly, after viewing more than 40 scenes and hundreds of individual objects, change detection performance after a 24-h delay remained well above chance.

In addition, recent studies have shown that these visual representations of individual objects are episodically organized into a larger representation in visual memory. Therefore, the availability of contextual information is capable of modulating visual object memory (Hollingworth, 2006, 2007; Jiang, Olson, \& Chun, 2000; Sun \& Gordon, 2009; Zimmer, 1998). For example, Zimmer used a delayed matching task to investigate whether the congruence of the spatial configuration (e.g., triangular or linear arrays) between study and test would affect visual shortterm memory (VSTM) for geometric figures and words. He found that participants' recognition performance was worse if the spatial configuration of the stimuli was changed from study to test, indicating that the spatial relations between items are corepresented in VSTM such that the congruence of spatial configuration between the study and test stimuli can affect VSTM performance. Similarly, Jiang et al. (2000) used a change detection paradigm to 
examine how items in VSTM are organized and related to each other. They observed that the invariance of the spatial configuration of the stimulus arrays between study and test affected their participants' ability to detect changes in an object's color, shape, or location. The results indicate that relational information between items is encoded in VSTM and that disruption of the original spatial configuration impairs the retrieval of target properties from VSTM. Recent studies in which real-world objects were used have demonstrated similar contextual effects (Hollingworth, 2006, 2007). For example, Hollingworth (2006) had participants view 3-D-rendered images of real-world scenes and tested their memory for the visual form of a target object presented either within the original scene context or in isolation. The results showed that object recognition performance was facilitated when the target objects were presented within the original scene context, demonstrating that object representations are episodically linked to the global scene context in memory so that context deletion impairs object recognition performance.

These results are consistent with the encoding specificity principle of memory, which suggests that memory retrieval is affected by the reinstantiation of the encoding context (Thomson \& Tulving, 1970; Tulving \& Thomson, 1973). For example, Thomson and Tulving (1970) had participants study cue-target pairs consisting of weakly associated words (e.g., train-BLACK) and tested them with a cued recall task. At test, the participants were given either the weakly associated cue (e.g., train) or a strongly associated cue (e.g., white) to assist recall of the target (e.g., BLACK). The weak cue was the same word (e.g., train) that had been paired with the target during study, whereas the strong cue was a different nonstudied word (e.g., white). The results showed that the participants recalled more targets in the reinstated weak cue condition than in the nonstudied strong cue condition, indicating that the weak cue was encoded with the target so that the reinstated weak cue serves as an effective retrieval cue for target recall. Although these earliest studies examining the encoding specificity principle focused primarily on retrieval of semantic information from long-term memory, the more recent studies reviewed above have shown that the same principle can be applied to nonverbal information retrieval from VSTM as well.

Given that a number of studies have demonstrated that encoding context affects subsequent visual memory retrieval, a relevant issue is what information is represented in visual memory. According to the encoding specificity principle, retrieval from memory is affected by the extent to which information relating the target and other items is encoded during study. Therefore, examining how different aspects of the encoding context affect visual memory is relevant to understanding the nature of the information represented in visual memory. For example, Jiang et al. (2000) suggested that the reinstatement of global spatial configuration is the key factor supporting visual memory retrieval regardless of information about the objects occupying those locations. Therefore, memory performance should be more accurate as long as the spatial layout remains constant across encoding and retrieval. However,
Hollingworth (2007, Experiment 8) reported that participants' change detection performance was impaired when nontarget objects exchanged locations within the original spatial configuration, demonstrating that maintenance of the abstract spatial layout alone is not sufficient to support memory retrieval. Information about the individual objects bound to those locations is important as well.

More recently, Sun and Gordon (2009) further demonstrated that contextual objects' identities and locations have independent effects on visual memory. In that study, Sun and Gordon had participants view various object arrays in a change detection task requiring memory for the visual form of a target object (e.g., orientation). Spatial and nonspatial properties of the array context were manipulated independently in the test image. The influence of spatial contextual information was examined by retaining the contextual objects' locations or by placing them in previously unoccupied locations, and the influence of nonspatial contextual information was examined either by retaining or by removing the contextual objects' identities (e.g., by blurring the objects or by replacing them with new objects). The results showed that change detection performance was more accurate when either contextual objects' location or identity was preserved in the test image. Therefore, after viewing arrays of objects, the objects' identities and locations are represented in visual memory and used to modulate subsequent visual memory performance.

In the present study, we further examined the fidelity of this contextual representation in visual memory. Previous studies have suggested that items in visual memory may have detailed representations, such as information regarding their identities and visual forms. However, it remains unclear what object features are stored as part of the contextual representation and play a role in modulating visual memory. In the Sun and Gordon (2009) study, contextual objects' identities were altered either by blurring or by replacing them with new objects. Such manipulations changed many nonspatial object features simultaneously; any or all of those features may have contributed to participants' change detection performance. Therefore, the goal of the present study was to replicate and extend the previous findings of Sun and Gordon by using the same task and design but manipulating individual visual features of contextual objects while their identities were held constant across study and test images. On each experimental trial, the participants saw two object arrays separated by a brief interstimulus interval, then were required to judge whether the orientation (Experiments 1A, 1B, and 2) or color (Experiments $3 \mathrm{~A}$ and $3 \mathrm{~B}$ ) of a target object was the same as it was in the initial array. The influence of object configuration was examined either by retaining or by changing the original spatial layout in the test image. We expected to replicate the previous finding of an independent influence of spatial configuration on visual memory. That is, the participants' change detection performance should be better if the original spatial layout remains the same in the test image. In addition, we examined the influence of different visual properties of the encoding context either by maintaining or by changing contextual objects' 
color (Experiments 1A, 1B, and 3A) or orientation (Experiments 2 and $3 \mathrm{~B}$ ). If contextual objects' color or orientation is stored as part of the contextual representation that plays a role in modulating visual memory, maintaining consistent object features between encoding and retrieval should facilitate target memory.

\section{EXPERIMENTS 1A AND 1B}

The purpose of Experiments 1A and 1B was to examine the effect of contextual information on visual object memory by manipulating contextual objects' location and color in a change detection task. A number of studies have shown that visual memory performance is impeded when contextual objects change their locations. However, relatively little is known about the effects of contextual objects' color on visual memory. Since color is an intrinsic property of an object's representation (e.g., Naor-Raz, Tarr, \& Kersten, 2003; Tanaka, Weiskopf, \& Williams, 2001), we were particularly interested in investigating the effect of changing contextual objects' color on visual memory in the present study. Two versions of color manipulation were used, in Experiments 1A and 1B, respectively.

\section{Method}

Participants. Forty-seven North Dakota State University undergraduates (27 in Experiment 1A, 20 in Experiment 1B) participated in the experiments for course credit. All of the participants had normal or corrected-to-normal vision and were naive to the purpose of the study.

Apparatus. The stimuli were displayed on a 17-in. CRT monitor with a refresh rate of $100 \mathrm{~Hz}$. Responses were collected through mouse clicks. The experiment was programmed using Presentation software (Neurobehavioral Systems, Albany, CA). The participants were tested individually in a room with normal interior lighting. The viewing distance was held constant at $90 \mathrm{~cm}$.

Stimuli. In Experiments 1A and 1B, 80 objects were selected from the Tarrlab Object Databank (M. J. Tarr, Carnegie Mellon Uni- versity, http://tarrlab.cnbc.cmu.edu). They were the same as those used by Sun and Gordon (2009). The screen was divided into an invisible $3 \times 4$ matrix, and 6 objects were randomly assigned to 6 of the 12 possible cells to create the object array stimuli. The entire array subtended $15.2^{\circ} \times 20.7^{\circ}$ with a white background. For the test images, the target object was postcued by a red square, and its orientation was either the same as it appeared in the initial array or mirror-reflected about its vertical axis. All of the contextual objects surrounding the target object either matched the study image or differed with respect to location, color, or both. That is, in the test image, the contextual objects either appeared in the same location or moved to previously unoccupied locations. In addition, in Experiment $1 \mathrm{~A}$, the contextual objects either retained their color or were replaced by an achromatic version of the original colored object. In Experiment 1B, the contextual objects either retained their color or had their color changed to a different color by varying the objects' hue while keeping constant the objects' luminance and saturation.

Procedure and Design. Each trial began with a fixation cross for $1,000 \mathrm{msec}$. The study image was then presented for $4,000 \mathrm{msec}$, followed by a blank screen for $900 \mathrm{msec}$ and then the test image. The test image remained visible until the participant responded. Feedback concerning accuracy was then provided for $400 \mathrm{msec}$ immediately after the response. Figure 1 illustrates the sequence of events in each trial.

These experiments followed a 2 (target orientation: same, different) $\times 2$ (contextual objects' location: same, different) $\times 2$ (contextual objects' color: same, different) factorial design. The participants completed a practice block of 16 trials and four experimental blocks of 40 trials each. The trials were presented in a random order for each participant. All eight conditions occurred equally often. This resulted in 20 observations for each of the eight conditions.

\section{Results}

In the present study, $A^{\prime}$ was used as an index of the participant's sensitivity to change (Grier, 1971). For each participant in each condition, $A^{\prime}$ was calculated using the mean hit rate when the target changed and the mean false alarm rate when it did not. The mean $A^{\prime}$ values for different conditions of Experiments $1 \mathrm{~A}$ and $1 \mathrm{~B}$ are shown in Tables 1A and 1B, respectively. The pattern of results was the same across the two experiments. Two-way (contextual

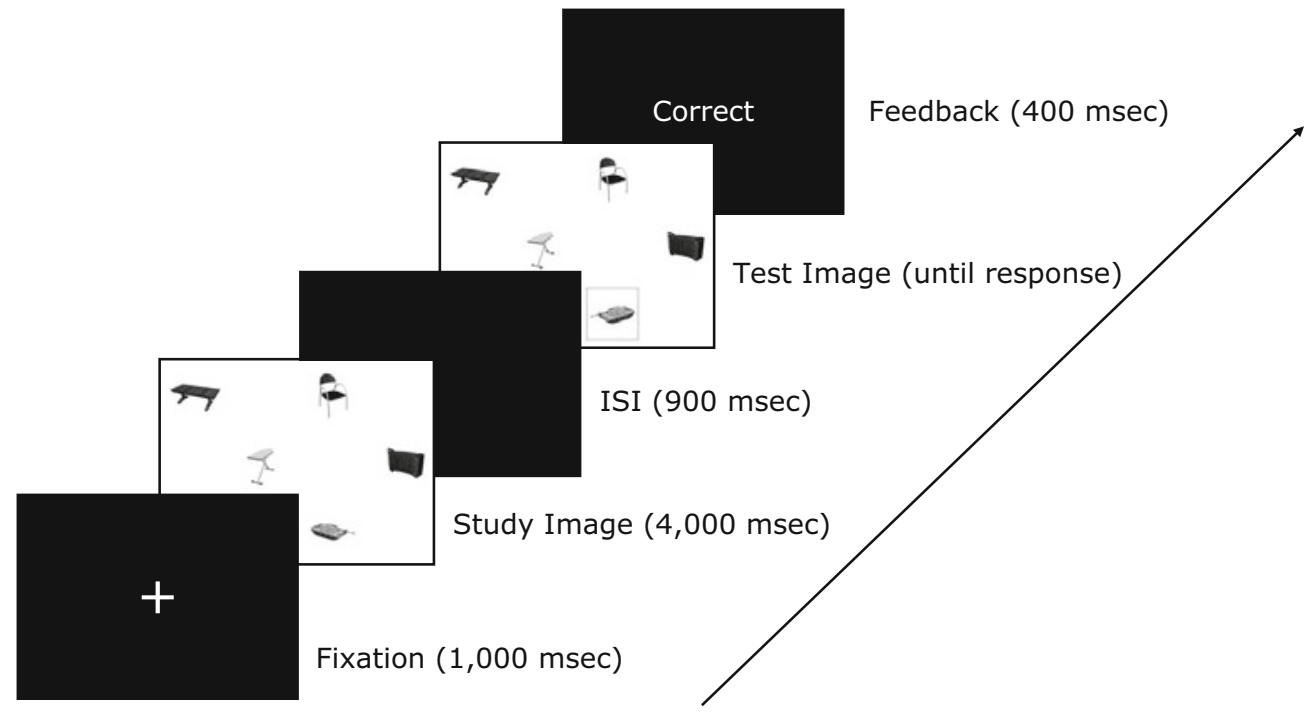

Figure 1. The sequence of events within a trial. In all of the experiments presented in this article, all stimuli were presented in color. ISI, interstimulus interval. 
Table 1A

Mean $A^{\prime}$ Data From Experiment 1A As a Function of Contextual Objects' Location and Color

\begin{tabular}{ccc}
\hline & \multicolumn{2}{c}{$\begin{array}{c}\text { Contextual } \\
\text { Objects'Location }\end{array}$} \\
\cline { 2 - 3 } $\begin{array}{c}\text { Contextual } \\
\text { Objects' Color }\end{array}$ & Same & Different \\
\hline Same & 0.85 & 0.78 \\
Achromatic & 0.84 & 0.79 \\
\hline
\end{tabular}

Table 1B

Mean $A^{\prime}$ Data From Experiment $1 B$ As a Function of Contextual Objects' Location and Color

Contextual

\begin{tabular}{ccc} 
Contextual & \multicolumn{2}{c}{$\begin{array}{c}\text { Contextual } \\
\text { Objects' Location }\end{array}$} \\
\cline { 2 - 3 } Objects' Color & Same & Different \\
\hline Same & 0.82 & 0.77 \\
Different & 0.83 & 0.78 \\
\hline
\end{tabular}

objects' location $\times$ contextual objects' color) ANOVAs conducted on the mean $A^{\prime}$ performance revealed a significant main effect of contextual objects' location [Experiment $1 \mathrm{~A}, F(1,26)=20.76, M S_{\mathrm{e}}=0.005, p=.000$; Experiment $\left.1 \mathrm{~B}, F(1,19)=12.26, M S_{\mathrm{e}}=0.004, p=.002\right]$. The main effect of contextual objects' color was not significant [Experiment 1A, $F(1,26)=0.000, M S_{\mathrm{e}}=0.003$, $p=.985$; Experiment $1 \mathrm{~B}, F(1,19)=1.879, M S_{\mathrm{e}}=0.002$, $p=.186]$. There was no interaction between contextual objects' location and color [Experiment 1A, $F(1,26)=$ 0.096; Experiment 1B, $F(1,19)=0.306]$.

To permit comparison of the present results with recent research on information retention in visual working memory, we also estimated the participants' memory capacity $(K)$ for this and later experiments. We computed $K$ using the formula initially developed by Pashler (1988) and later refined by Cowan (2001). The formula is $K=S \times(H-$ $F$ ), where $K$ is the memory capacity, $S$ is the set size of the array, $H$ is the hit rate, and $F$ is the false alarm rate. The mean $K$ values for Experiments 1A and 1B were 2.7 and 2.5 , respectively. These capacity estimates are similar to those previously reported for complex visual objects (e.g., Alvarez \& Cavanagh, 2004).

\section{Discussion}

On the basis of these results, three main points can be made. First, overall change detection performance was well above chance, confirming that detailed object representations can be maintained after viewing arrays of objects. Second, performance was modulated by changes in the locations of contextual objects, suggesting that object representations in visual memory are linked to each other such that contextual information can affect visual memory for individual objects. Finally, despite its importance to object representation (e.g., Naor-Raz et al., 2003), contextual object color is not an effective cue for retrieval from visual object memory. The implications of this finding will be addressed in the General Discussion section. Because we were interested in examining how different aspects of an object's context affect visual memory, we next conducted an experiment to examine how visual memory performance is affected by changes in a different object feature-namely, the object's orientation.

\section{EXPERIMENT 2}

Recent studies in which object representations in transsaccadic memory were examined suggest that detailed representations of object orientation can be encoded during a preview and affect subsequent object identification (e.g., Gordon, Vollmer, \& Frankl, 2008; Henderson \& Siefert, 2001). Moreover, research has shown that changes to elongated objects' orientations impair location change detection performance (e.g., Jiang, Chun, \& Olson, 2004, Experiment 1). Together, these findings suggest that object orientation may play an influential role in modulating visual memory. Therefore, Experiment 2 was conducted to examine how contextual objects' orientation and the global array configuration affect visual memory performance. The participants were asked to perform a change detection task in which both contextual objects' location and their orientation were manipulated in the test image. We were interested in whether these different properties of the context would affect visual memory independently.

\section{Method}

Participants. Twenty-three undergraduates from North Dakota State University completed the experiment for course credit. All of the participants had normal or corrected-to-normal vision. None had participated in the previous experiments, and all of them were naive to the purpose of the study.

Apparatus, Stimuli, Procedure, and Design. Experiment 2 was identical to Experiments 1A and 1B, except that contextual objects' orientation, rather than color, was manipulated to examine the effect of nonspatial contextual information. From the study to the test image, these contextual objects either maintained their orientation or were presented in the mirror-reversed orientation. Figure 2 shows sample stimuli and manipulations in different conditions.

\section{Results}

Table 2 shows the mean $A^{\prime}$ performance in each of the experimental conditions. A two-way (contextual objects' location $\times$ contextual objects' orientation) ANOVA conducted on the mean $A^{\prime}$ performance showed a significant main effect of the contextual objects' location $[F(1,22)=$ $\left.5.61, M S_{\mathrm{e}}=0.007, p=.027\right]$, as well as a significant main effect of the contextual objects' orientation $[F(1,22)=$ $\left.6.85, M S_{\mathrm{e}}=0.005, p=.016\right]$. There was no interaction between the contextual objects' location and their orientation $[F(1,22)<1]$. Finally, the estimated memory capacity $(K)$ was 2.6 for Experiment 2.

\section{Discussion}

As in Experiments 1A and 1B, the participants' overall change detection performance was well above chance, indicating that detailed object representations can be maintained in visual memory. Moreover, the participants' change detection performance was worse when the array configuration changed from study to test, demonstrating the contribution of spatial layout to visual memory. In addition, the results showed that changes to the contextual objects' orientation decreased the participants' change detection performance, 


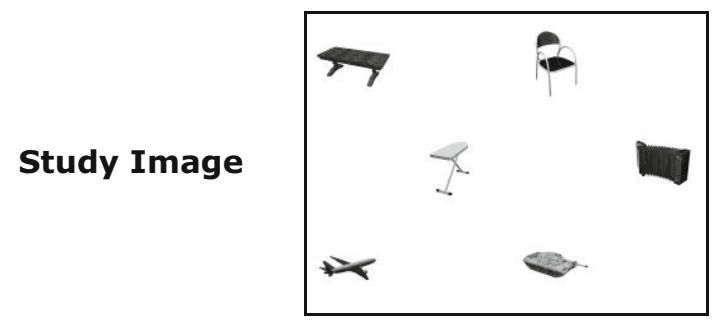

A

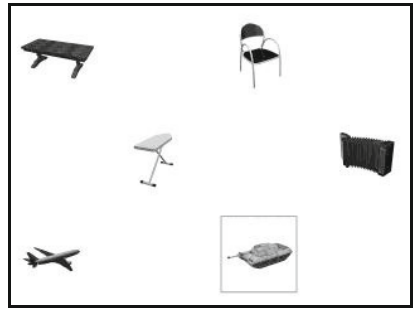

B

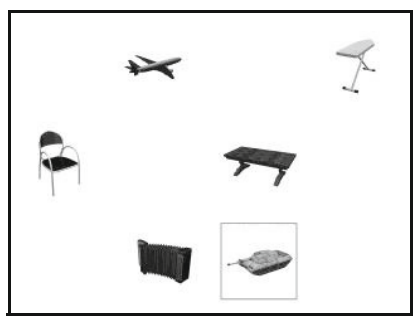

C

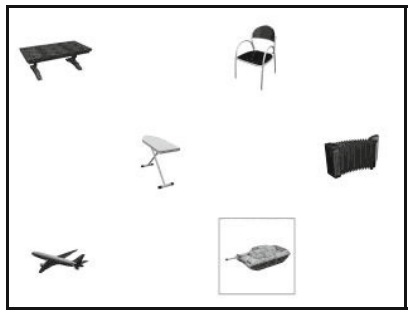

D

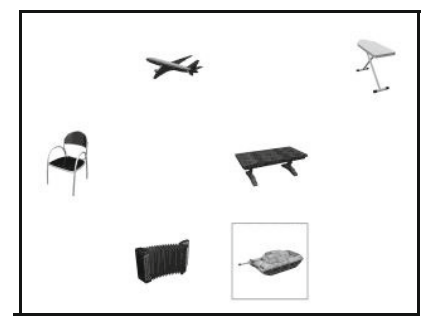

Figure 2. Sample study and test images in Experiment 2. All test images depict trials in which the target object has changed orientation. In the experiment, all stimuli were presented in color. (A) A sample test image used in the same-location and same-orientation condition; (B) a sample test image used in the different-location and same-orientation condition; (C) a sample test image used in the same-location and different-orientation condition; (D) a sample test image used in the different-location and differentorientation condition.

indicating that representation of object orientation plays a role in modulating visual memory as well.

\section{EXPERIMENTS 3A AND 3B}

The results of Experiments 1A, 1B, and 2 showed that visual memory for a target object's visual form (e.g., orientation) was consistently affected by contextual objects'

Table 2

Mean $A^{\prime}$ Data From Experiment 2 As a Function of Contextual Objects' Location and Orientation

\begin{tabular}{ccc}
\hline \multirow{2}{*}{$\begin{array}{c}\text { Contextual } \\
\text { Objects' Orientation }\end{array}$} & \multicolumn{2}{c}{$\begin{array}{c}\text { Contextual } \\
\text { Objects' Location }\end{array}$} \\
\cline { 2 - 3 } & Same & Different \\
\hline Same & 0.84 & 0.79 \\
Mirror reversed & 0.79 & 0.76 \\
\hline
\end{tabular}

locations at test, replicating previous research that shows the influence of spatial configuration on visual memory. In addition, contextual objects' orientation, but not color, was able to affect target memory. This raises a question about whether the influences of contextual objects' visual features on visual memory is completely stimulus driven or can be modulated by selective attention. On the basis of a bottom-up perspective, for example, it is possible that object orientation is obligatorily represented and used to modulate visual object memory. However, it is also plausible that the goal of the current task may affect the role of different visual features in modulating subsequent visual memory performance, which would explain why there were different influences of contextual objects' orientation and color on target memory in an orientation change detection task. Consequently, contextual objects' orientation, rather than color, serves as a better retrieval cue, because it is preferentially represented and used to affect 
target memory in an orientation change detection task. If this is the case, it is reasonable to assume that object color, rather than orientation, would serve as an effective memory retrieval cue when it is required by the current task.

The purpose of Experiments 3A and 3B, therefore, was to test whether attention to a specific object feature would enhance its role in modulating visual object memory. We examined this question by replacing the previous orientation change detection task with a color change detection task, in which participants had to determine whether a target object's color changed after they viewed the study image. Similar to the manipulations of our first two experiments, the effect of spatial configuration was tested by maintaining or changing contextual objects' locations between the study and test images. Moreover, the influences of the contextual objects' color and orientation on the participants' color change detection performance were examined in Experiments 3A and 3B, respectively.

\section{Method}

Participants. Forty-two North Dakota State University undergraduates (21 in Experiment 3A, 21 in Experiment 3B) participated in the experiments for course credit. All of the participants had normal or corrected-to-normal vision and normal color vision. None had participated in Experiments 1A, 1B or 2, and all of them were naive to the purpose of the study.

Apparatus, Stimuli, Procedure, and Design. Except for the task requirement and stimulus generation described below, Experiments $3 \mathrm{~A}$ and $3 \mathrm{~B}$ were identical to Experiments $1 \mathrm{~A}, 1 \mathrm{~B}$, and 2, respectively. First (in Experiment 3A), participants were asked to perform a color change detection task rather than an orientation change detection task. That is, participants had to report whether the color of a target object was the same as it appeared during initial viewing, or different. Second (in Experiment 3B), twenty 3-D-rendered images of real-world objects, rather than the objects chosen from the Tarrlab

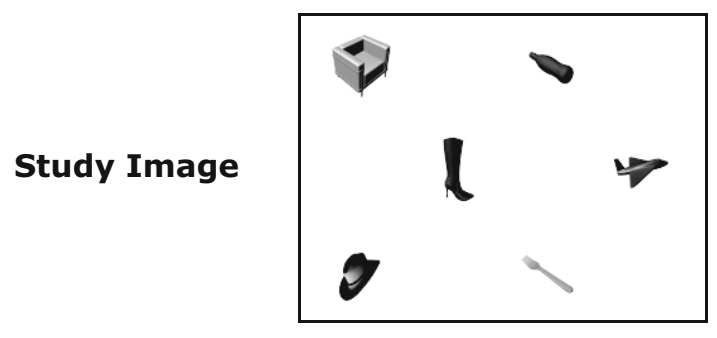

A

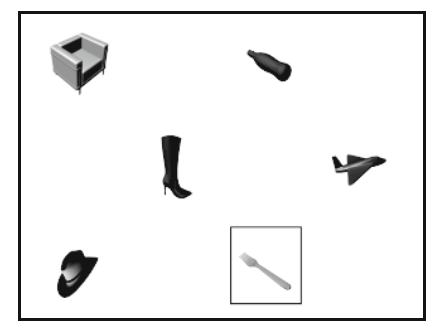

Test Images

B

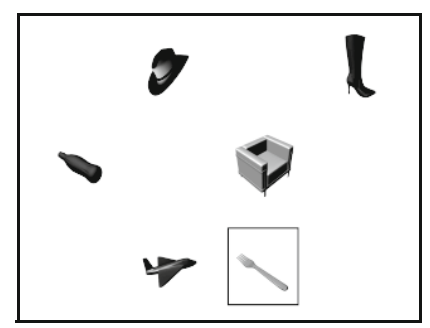

C

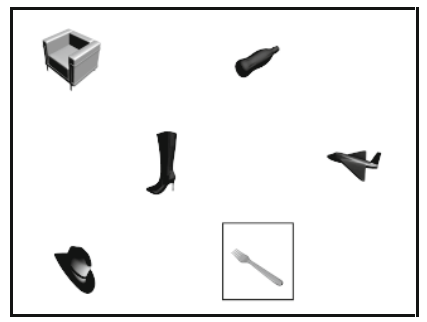

D

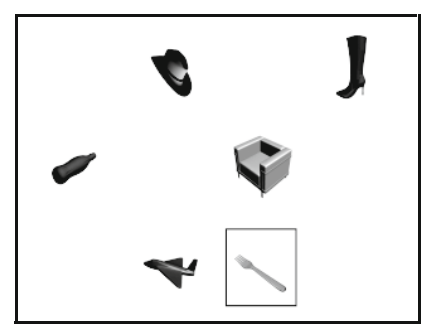

Figure 3. Sample study and test images in Experiment 3B. All test images depict trials in which the color of a target object remained the same as it appeared in the study image. In the experiment, all stimuli were presented in color. (A) A sample test image used in the same-location and same-orientation condition; (B) a sample test image used in the different-location and same-orientation condition; (C) a sample test image used in the same-location and different-orientation condition; (D) a sample test image used in the differentlocation and different-orientation condition. 
Object Databank, that were collected from various Web sites. None of the objects had a typical or diagnostic color (e.g., yellow banana or orange pumpkin). In addition, four different color versions were created for each of the twenty objects by using the Maya 3-D modeling software (Autodesk, San Rafael, CA). Figure 3 shows sample stimuli and manipulations in different conditions in Experiment 3B.

\section{Results}

The mean $A^{\prime}$ values for the different experimental conditions of Experiments $3 \mathrm{~A}$ and $3 \mathrm{~B}$ are shown in Tables $3 \mathrm{~A}$ and $3 \mathrm{~B}$, respectively. For Experiment $3 \mathrm{~A}$, a two-way (contextual objects' location $\times$ contextual objects' color) ANOVA conducted on the mean $A^{\prime}$ performance showed significant main effects for contextual objects' location $\left[F(1,20)=19.56, M S_{\mathrm{e}}=0.003, p=.000\right]$ and for contextual objects' color $\left[F(1,20)=13.54, M S_{\mathrm{e}}=0.004, p=\right.$ $.001]$. The interaction between contextual objects' location and color was not significant $[F(1,20)=0.049]$. For Experiment $3 \mathrm{~B}$, the results of a two-way (contextual objects' location $\times$ contextual objects' orientation) ANOVA showed a significant main effect for contextual objects' location $\left[F(1,20)=12.72, M S_{\mathrm{e}}=0.004, p=.002\right]$ but not for contextual objects' orientation $[F(1,20)=0.31$, $\left.M S_{\mathrm{e}}=0.003, p=.584\right]$. There was no interaction between contextual objects' location and orientation $[F(1,20)=$ $0.133]$. Finally, the mean $K$ values for Experiments $3 \mathrm{~A}$ and $3 \mathrm{~B}$ were 2.8 and 3.2 , respectively.

\section{Discussion}

The results of Experiments $3 \mathrm{~A}$ and $3 \mathrm{~B}$ support the idea that detailed object representations can be maintained in visual memory, since the participants' mean $A^{\prime}$ scores were well above chance across different experimental conditions. In addition, changes to the array configuration in the test image resulted in lower change detection performance, demonstrating the robustness of the effect of spatial contextual information in visual memory. Finally, the results of Experiments $3 \mathrm{~A}$ and $3 \mathrm{~B}$ showed that contextual objects' color, but not their orientation, had an effect on the participants' performance in detecting changes to a target object's color. This result suggests that orientation information is not included in the contextual represen-

Table 3A

Mean $A^{\prime}$ Data From Experiment 3A As a Function of Contextual Objects' Location and Color

Contextual

\begin{tabular}{ccc} 
Contextual & \multicolumn{2}{c}{$\begin{array}{c}\text { Contextual } \\
\text { Objects'Location }\end{array}$} \\
\cline { 2 - 3 } Objects' Color & Same & Different \\
\hline Same & 0.86 & 0.81 \\
Different & 0.80 & 0.76 \\
\hline
\end{tabular}

Table 3B

Mean $A^{\prime}$ Data From Experiment 3B As a Function of Contextual Objects' Location and Orientation

\begin{tabular}{ccc}
\hline \multirow{2}{*}{$\begin{array}{c}\text { Contextual } \\
\text { Objects' Orientation }\end{array}$} & \multicolumn{2}{c}{$\begin{array}{c}\text { Contextual } \\
\text { Objects' Location }\end{array}$} \\
\cline { 2 - 3 } & Same & Different \\
\hline Same & 0.86 & 0.81 \\
Mirror reversed & 0.86 & 0.82 \\
\hline
\end{tabular}

tations that facilitate change detection in a color change detection task, demonstrating that selective attention plays an important role in determining the representations of object visual features in visual memory.

\section{GENERAL DISCUSSION}

The primary goal of this study was to investigate how spatial configuration and individual visual features of objects influence visual object memory by using a series of change detection experiments. An orientation change detection task was used in Experiments 1A, 1B, and 2, whereas a color change detection task was used in Experiments $3 \mathrm{~A}$ and $3 \mathrm{~B}$. On each experimental trial, after viewing an object array, the participants had to discriminate whether a target object's visual information (e.g., orientation, color) had changed in the test image. In the test image, the contextual objects' locations and visual features were manipulated so that their influences on the participants' change detection performance could be compared later. The results of five experiments showed that the participants were able to detect changes to target objects' surface features on a significant proportion of trials. Moreover, change detection performance was better when the contextual objects' location remained the same from study to test. Finally, the participants' orientation change detection performance was affected by changing the contextual objects' orientation, but not their color (Experiments $1 \mathrm{~A}, 1 \mathrm{~B}$, and 2 ), whereas color change detection performance was affected by the contextual objects' color, rather than their orientation (Experiments 3A and 3B).

The present study confirms that robust visual representations of objects can be retained after viewing complex images (e.g., an array of six objects), supporting previous findings that have shown that detailed object representations can be generated and maintained in visual memory (e.g., Hollingworth, 2005; Hollingworth \& Henderson, 2002; Sun \& Gordon, 2009). Furthermore, our results showed that the participants' change detection performance was higher when the original object array was preserved at test, demonstrating that visual object memory can be modulated by contextual information. The results are in accord with those of previous studies suggesting that individual items are wedded to a more extensive representation in memory; hence, contextual congruence between study and test images affects subsequent visual object memory performance (e.g., Hollingworth, 2006, 2007; Jiang et al., 2000; Sun \& Gordon, 2009; Zimmer, 1998). As the encoding specificity principle suggests, reappearance of the encoding context serves as an effective retrieval cue, facilitating memory performance (Thomson \& Tulving, 1970; Tulving \& Thomson, 1973).

Given that encoding context affects subsequent visual memory retrieval, it is important to examine how different aspects of this context affect visual memory. This issue is relevant to understanding what information is represented in visual memory and the resolution of the object representations in visual memory. A number of studies have demonstrated that visual object memory is affected by contextual objects' location (Hollingworth, 2007; Jiang 
et al., 2000; Sun \& Gordon, 2009). For example, Jiang et al. (2000) reported that the congruence of spatial configuration between study and test modulates visual memory for individual visual items, regardless of information about the items occupying those locations. Similar results were found in studies in which the effects of contextual cuing were examined (Chun, 2000; Chun \& Jiang, 1998). For example, Chun and Jiang reported that visual attention can be quickly guided to a target when the spatial configuration of the search array has been repeated several times. Moreover, as long as the global spatial configuration of the search array is maintained, this contextual cuing effect can be obtained even when distractors' identities change. In summary, these studies suggest that this contextual representation is purely spatial, coding object locations but not necessarily detailed identity information.

The present study supports the hypothesis that spatial configuration plays an influential role in modulating visual object memory, because the results of five experiments consistently showed that change detection performance was better when contextual objects' location remained the same from study to test. However, our results further demonstrate that changes in contextual objects' visual features impair visual memory, even when their spatial location is held constant. Therefore, an abstract representation of spatial configuration is not alone in affecting visual memory retrieval; information about the individual objects that make up the configuration is important as well. This finding is consistent with recent findings showing that object information is important for supporting visual memory performance (Hollingworth, 2007; Sun \& Gordon, 2009). For example, Hollingworth (2007) manipulated the binding of contextual objects to locations and revealed that a contextual representation preserves not only the spatial configuration, but also information about the objects bound to each location. In addition, Sun and Gordon demonstrated that either contextual objects' location or identity is capable of modulating visual memory independently. Nevertheless, our present results extend this previous work by demonstrating that different visual properties of contextual objects are capable of modulating visual memory as well.

The present results further suggest, however, that not every visual feature that makes up the original context can affect visual memory. For example, the results of Experiments $1 \mathrm{~A}, 1 \mathrm{~B}$, and 2 showed that the participants' ability to detect an orientation change in the target object was affected by contextual objects' orientation but not their color. The observed effect of contextual objects' orientation, but not their color, on the participants' performance in detecting changes in target objects' orientations parallels previous findings showing how changes to contextual items' orientation, rather than color, influence location change detection performance (Jiang et al., 2004; Jiang et al., 2000). Moreover, recent studies in which the nature of object file representations was examined have shown that object orientation information is preserved in transsaccadic memory and therefore plays an influential role in preserving object continuity (e.g., Gordon et al., 2008; Henderson \& Siefert, 2001). In a recent study, Gordon and Vollmer (2010) further showed that the influence of object color on pre- serving object continuity is modulated by object-color associations. That is, changes to target objects' color disrupt object continuity across saccades, but only for objects that have typical colors (e.g., a yellow banana). In addition, note that research on contextual cuing has shown that color does not play an important role in facilitating visual search in real-world scenes, even when color is a reliable cue to target location (Ehinger \& Brockmole, 2008). Therefore, the results of Experiments 1A, 1B and 2 are in line with recent studies showing that object orientation, but not color, reliably affects memory performance.

However, this finding raises an interesting question about whether the influence of contextual objects' orientation on visual object memory is completely stimulus driven or whether it can be modulated by top-down attention. That is, if orientation information is obligatorily encoded and retrieved to modulate visual memory, change detection performance should be affected by contextual objects' orientation, despite the current task requirement. The results of Experiments $3 \mathrm{~A}$ and $3 \mathrm{~B}$, however, showed that contextual objects' color, but not their orientation, significantly affected the participants' color change detection performance. Therefore, the results suggest that selective attention plays a significant role in modulating the effects of object features on visual object memory. Task-relevant features may therefore be preferentially attended and used to assist visual object memory retrieval.

Combined, the results of the present experiments indicate that the influence of different aspects of nonspatial context (e.g., object orientation and color) on visual object memory is significantly modulated by selective attention. Consequently, orientation change detection performance is affected by contextual objects' orientation but not their color (Experiments 1A, 1B, and 2), whereas color change detection performance is affected by contextual objects' color but not their orientation (Experiments $3 \mathrm{~A}$ and $3 \mathrm{~B}$ ). This finding is consistent with those of recent studies showing that participants can control which information is encoded and held in visual working memory on the basis of the goals of their current task (e.g., Droll, Hayhoe, Triesch, \& Sullivan, 2005; Vogel, McCollough, \& Machizawa, 2005; Woodman \& Vogel, 2008). For example, Woodman and Vogel used a masked change detection paradigm to examine the encoding rates of different visual features of colored and oriented rectangles. Specifically, in different experimental blocks, they instructed participants to remember only the color, only the orientation, or both features for each object. That is, the sample stimuli were maintained identically across different blocks and only the instructions as to which features were task relevant and the types of possible changes differed. If object features can be selectively encoded according to the task goals, different encoding rates should be obtained for different conditions. Woodman and Vogel's results supported this assumption and showed distinct encoding rates for object color and orientation. A subsequent event-related potential study in which the amplitude of the contralateral delay activity was examined further confirmed that only task-relevant features are encoded and maintained in visual working memory (Woodman \& Vogel, 2008). 
Note, however, that task relevance alone does not completely determine what visual features are represented in visual memory and their influence on subsequent visual memory retrieval. Recent research has shown that taskirrelevant features cannot be effectively ignored under certain conditions, such as the orientation of elongated objects in a location change detection task (e.g., Jiang et al., 2004). Moreover, Jiang and Leung (2005) found that previously ignored contexts facilitate visual search speed when they later become the attended contexts, demonstrating that the visual system is able to track both task-relevant and -irrelevant information for better future performance. Therefore, we cannot conclude that only task-relevant object features are encoded and represented in visual memory. The question about the fate of task-irrelevant aspects of a context requires further investigation.

\section{CONCLUSION}

Visual objects are composed of multiple features, such as color and orientation. Moreover, objects tend to occupy distinct locations in visual space. After viewing complex visual stimuli, such as an array of objects, a detailed contextual representation can be maintained in visual memory and used to modulate retrieval of related information from visual memory. Our results suggest that both spatial configurations and object features are preserved in this larger representation. In addition, both types of contextual information can be used to assist visual memory performance. Therefore, disrupting the original spatial configuration impairs participants' performance in detecting changes to target objects' visual properties. Compared with the robust effect of spatial layout on target memory, however, our results suggest that the effect of various visual features on visual memory is significantly modulated by selective attention. Therefore, changes to contextual objects' orientation but not their color, impair orientation change detection performance, whereas changes to contextual objects' color but not their orientation, reduce color change detection performance.

\section{AUTHOR NOTE}

This project was supported by Grant 1P20 RR020151 from the National Center for Research Resources, a component of the National Institutes of Health. The project was also supported by the National Science Foundation Grant BCS-0443998. We thank James Brockmole, David Waller, Carrick Williams, and one anonymous reviewer for their helpful comments on the manuscript. Correspondence concerning this article should be addressed to H.-M. Sun, Department of Psychology, North Dakota State University, Fargo, ND 58105 (e-mail: mei.sun@ndsu.edu).

\section{REFERENCES}

Alvarez, G. A., \& Cavanagh, P. (2004). The capacity of visual short term memory is set both by visual information load and by number of objects. Psychological Science, 15, 106-111.

Chun, M. M. (2000). Contextual cueing of visual attention. Trends in Cognitive Sciences, 4, 170-178.

Chun, M. M., \& Jiang, Y. (1998). Contextual cueing: Implicit learning and memory of visual context guides spatial attention. Cognitive Psychology, 36, 28-71

Cowan, N. (2001). The magical number 4 in short-term memory: A reconsideration of mental storage capacity. Behavioral \& Brain Sciences, 24, 87-114.
Droll, J. A., Hayhoe, M. M., Triesch, J., \& Sullivan, B. T. (2005). Task demands control acquisition and storage of visual information. Journal of Experimental Psychology: Human Perception \& Performance, 31, 1416-1438.

Ehinger, K. A., \& Brockmole, J. R. (2008). The role of color in visual search in real-world scenes: Evidence from contextual cuing. Perception \& Psychophysics, 70, 1366-1378.

Gordon, R. D., \& Vollmer, S. D. (2010). Episodic representation of diagnostic and non-diagnostic object colour. Visual Cognition, 18, 728-750.

Gordon, R. D., Vollmer, S. D., \& FrankL, M. L. (2008). Object continuity and the transsaccadic representation of form. Perception \& Psychophysics, 70, 667-679.

GRIER, J. B. (1971). Nonparametric indexes for sensitivity and bias: Computing formulas. Psychological Bulletin, 75, 424-429.

Henderson, J. M., \& Siefert, A. B. C. (2001). Types and tokens in transsaccadic object identification: Effects of spatial position and left-right orientation. Psychonomic Bulletin \& Review, 8, 753-760.

HollingWORTH, A. (2004). Constructing visual representations of natural scenes: The roles of short- and long-term visual memory. Journal of Experimental Psychology: Human Perception \& Performance, 30, 519-537.

HoLLingworTh, A. (2005). The relationship between online visual representation of a scene and long-term scene memory. Journal of Experimental Psychology: Learning, Memory, \& Cognition, 31, 396-411.

Hollingworth, A. (2006). Scene and position specificity in visual memory for objects. Journal of Experimental Psychology: Learning, Memory, \& Cognition, 32, 58-69.

HollingWORTH, A. (2007). Object-position binding in visual memory for natural scenes and object arrays. Journal of Experimental Psychology: Human Perception \& Performance, 33, 31-47.

Hollingworth, A., \& Henderson, J. M. (2002). Accurate visual memory for previously attended objects in natural scenes. Journal of Experimental Psychology: Human Perception \& Performance, 28, 113136.

Hollingworth, A., Williams, C. C., \& Henderson, J. M. (2001). To see and remember: Visually specific information is retained in memory from previously attended objects in natural scenes. Psychonomic Bulletin \& Review, 8, 761-768.

JiAng, Y., Chun, M. M., \& Olson, I. R. (2004). Perceptual grouping in change detection. Perception \& Psychophysics, 66, 446-453.

JiANG, Y., \& LEUNG, A. W. (2005). Implicit learning of ignored visual context. Psychonomic Bulletin \& Review, 12, 100-106.

JiAnG, Y., Olson, I. R., \& ChUn, M. M. (2000). Organization of visual short-term memory. Journal of Experimental Psychology: Learning, Memory, \& Cognition, 26, 683-702.

NAOR-RAZ, G., TARR, M. J., \& Kersten, D. (2003). Is color an intrinsic property of object representation? Perception, 32, 667-680.

PASHLER, H. (1988). Familiarity and visual change detection. Perception \& Psychophysics, 44, 369-378.

Sun, H.-M., \& Gordon, R. D. (2009). The effect of spatial and nonspatial contextual information on visual object memory. Visual Cognition, 17, 1259-1270.

TANaKa, J. W., Weiskopf, D., \& Williams, P. (2001). The role of color in high-level vision. Trends in Cognitive Sciences, 5, 211-215.

Thomson, D. M., \& Tulving, E. (1970). Associative encoding and retrieval: Weak and strong cues. Journal of Experimental Psychology, 86, 255-262

Tulving, E., \& Thomson, D. M. (1973). Encoding specificity and retrieval processes in episodic memory. Psychological Review, 80, 352-373.

Vogel, E. K., McCollough, A. W., \& Machizawa, M. G. (2005). Neural measures reveal individual differences in controlling access to working memory. Nature, 438, 500-503.

Woodman, G. F., \& Vogel, E. K. (2008). Selective storage and maintenance of an object's features in visual working memory. Psychonomic Bulletin \& Review, 15, 223-229.

Zimmer, H. D. (1998). Spatial information with pictures and words in visual short-term memory. Psychological Research, 61, 277-284.

(Manuscript received August 12, 2009; revision accepted for publication April 23, 2010.) 